\title{
EVOLUCIÓN DEL MODELO DE RESPONSABILIDAD SOCIAL EMMPRESARIAL
}

\author{
EVOLUTION OF THE MODEL OF \\ CORPORATE SOCIAL RESPONSIBILITY
}

Miguel A. Bustamante $U^{1,2}$

1 Profesor Investigador Invitado. Facultad de Especialidades Eempresariales, Universidad Católica de Santiago de Guayaquil, Av. Carlos Julio Arosemena
Km. 11⁄2 vía Daule, Guayaquil, Ecuador.
2 Facultad de Economía y Negocios, Universidad de Talca, Campus Lircay. Avenida Lircay S/N, Talca, Chile.

RESUMEN

ABSTRACT

Este artículo presenta un estudio de la evolución de un modelo de medición de la responsabilidad social empresarial para un conjunto de empresas chilenas que busca interpretar, mediante la comparación de las preocupaciones sociales y empresariales en dos momentos de análisis: Se identifica la evolución de la estructura inicial y actualizada de variables relevantes representativas de beneficios o costos sociales que impactan la sociedad. Se analizan dos modelos de gestión empresarial en término de su evolución analizando los indicadores estadísticos, prioridades y valores estandarizados, que refieren el cambio ocurrido entre dos momentos de las preocupaciones socio económicas, empresariales y sociales, determinadas desde una perspectiva ética de análisis. El estudio pone de manifiesto el cambio ocurrido entre estos dos momentos, lo cual se pone en evidencia mediante las valoraciones sociales de grupos de variables o dimensiones, variables e indicadores más y menos relevantes para dar cumplimiento a la responsabilidad social de las organizaciones.

PALABRAS CLAVE: ética de negocios; responsabilidad social; responsabilidad empresarial; balance de responsabilidad social empresarial

This article presents a study of the evolution of a measurement model of corporate social responsibility for a group of Chilean companies that seeks to interpret, by comparing social and business concerns in two moments of analysis: The evolution of the structure is identified initial and updated of relevant variables that represent benefits or social costs that impact society. Two business management models are analyzed in terms of their evolution by analyzing the statistical indicators, priorities and standardized values, which refer to the change that occurred between two moments of socio-economic, business and social concerns, determined from an ethical perspective of analysis. The study reveals the change that occurred between these two moments, which is evidenced by social assessments of groups of variables or dimensions, variables and indicators that are more and less relevant to comply with the social responsibility of organizations.

KEYWORD: business ethics; social responsibility; corporate responsibility; corporate social responsibility balance 


\section{Introducción}

El enfoque ético del hacer empresarial (Chevalier, 1978) acentúa el necesario carácter social de las empresas (Chiappe y Consigilio, 2016), considerando lo económico como parte constitutivo de lo social, como base ética de las decisiones organizacionales (Freije, 1996).

Es por ello que se reconocen las dificultades inherentes a la medición de lo social (Satién, 1993) lo que resulta complejo y equivalente a la medición de lo simplemente humano que puede afectar a la comunidad en general y a las empresas en particular, ralentizando las opción estratégicas factibles de ser sostenidas mediante los resultados de un modelo de valoración que, efectivamente, ponga en evidencia su contribución social (Castillo y Parragué, 2008).

\section{Sociabilidad humana}

No es fácil definir con precisión conceptos tales como social, sociedad o sociabilidad. (Rodriguez Luño 1984), pero es claro que comprenden al menos tres elementos distinguibles (Widow, 1988), a decir: pluralidad, unión y convivencia. Pluralidad, porque un individuo no forma una sociedad consigo mismo; unión, porque tampoco una pluralidad de individuos independientes da lugar a una sociedad; y convivencia porque no cualquier tipo de relación es social (Quispe, 2015).

La sociedad como sistema complejo, es propia y natural a la raza humana (Bustamante, 2004). Es por ello que, muchas de las disciplinas científicas que se han abocado al estudio de los temas históricos, sociológicos, antropológicos y etnológicos, han llegado a una conclusión unívoca: El ser humano existe siempre en relación con otros seres humanos (Capra, 1982; Widow, 1988), lo que equivale a una permanente interacción entre ellos (David Baron, 2011 a,b; 2016). Efectivamente, el hombre vive, trabaja, descansa, fomenta la cultura y los valores del espíritu en estrecha unión con sus semejantes (Flores, 1994). A este respecto Aristóteles en Política, Libro 1, planteó: El individuo que permanece intencionalmente fuera de la sociedad, es un ser degradado o un ser superior a la especie humana, algo menos o algo más que un hombre, pero no un hombre. (Aristóteles, 1992)

La sociedad no es sujeto de vida propia, como es el hombre, porque no hay vida de la sociedad equivalente a la vida de los individuos (Widow, 1988). Las únicas realidades humanas sustantivas (Verdugo M, 1998) y las únicas que viven en el sentido genuino de la palabra vivir, son las personas individuales, (Verdugo y García, 1998) quienes nacen, crecen y mueren en la sociedad. En sociedad el hombre satisface necesidades básicas de seguridad, afecto, jerarquía, integración, progreso, etc. El hombre vive naturalmente en sociedad porque allí se provee la satisfacción de sus necesidades y, puesto que, está en relación con otros hombres, da origen a una convivencia eminentemente social. Esta convivencia no puede prescindir de un orden y por tanto surge una organización política para esta sociedad (Widow, 1988). La sociedad en consecuencia, se presenta entonces no como un producto artificial y voluntario del hombre, sino, como el modo específico de vivir del hombre, puesto que habrá de existir "más ecología en la economía de mercado, y más economía de mercado en la ecología..." (Vohre, 1998).

\section{Dimensiones fundamentales de la responsabilidad empresarial}

Es preciso hacer una breve referencia a los conceptos éticos que condicionan, en cierto modo, a la empresa (Parra, 1980), los cuales se expresan en que la empresa es un ente orgánico e institucional creado por el hombre y cuya acción creadora cabe dentro de lo propiamente humano (Widow, 1988). El quehacer humano cualificado y el esfuerzo consciente del individuo orientado a transformar el mundo, reciben necesariamente el sello e influencia de su personalidad (Battaglia, et al., 2014).

El desarrollo de la empresa es necesario y deseable, puesto que la experiencia ha comprobado que está vinculada estrechamente a la evolución y al destino humano (Widow, 1988). La existencia de la empresa es vital, de modo que en ella allí reside su principal compromiso social, de manera que el uso y asignación de recursos a fines que no sean estos (Friedman, 1988 , pueden comprometer la existencia misma de la empresa y por consiguiente, restar a la sociedad de los beneficios de su existencia (Battaglia, et al., 2014).

\section{Paradigma Estadístico}

La teoría general de la administración ha recibido en el transcurso de los últimos 30 años una infinidad de contribuciones de la estadística bajo la forma de modelos (Chiavenato I., 1987) capaces de proporcionar soluciones instrumentales a los problemas empresariales, ya sea en el área de recursos humanos, como en la producción, comercialización, finanzas o en la propia área de administración general (Hiil y Jones, 2005).

Un modelo (Chiavenato I., 1987) puede ser usado como medio de simulación de situaciones futuras, así como para la evaluación de la probabilidad de ocurrencia de acciones sujetas al azar, de forma tal que proporcione datos suficientes que permitan medir el impacto y alcance de una situación futura con una razonable esperanza de ocurrencia (Amato, 2015). 
Por su parte, el llamado modelo matemático o estadístico ofrece innumerables ventajas, (Chiavenato I., 1987). En este caso, es posible mencionar que un modelo matemático permite descubrir y entender los hechos que representa y que sintetizan una situación dada (Freiberg, et al., 2013), todo ello, mejor de lo que lo permitiría una descripción verbal (Rosa, 2013). Un modelo, en consecuencia, facilita el descubrimiento de relaciones existentes entre diversos aspectos de un problema, que no necesariamente se expresan por sí solos en una descripción verbal.

Por otra parte, un modelo matemático permite tratar un problema en su conjunto y considerar todas las variables principales simultáneamente, siendo por ello susceptible de ampliación por etapas, hasta llegar a incluir factores abandonados en la descripción verbal (Freiberg, et al., 2013). En general, su ventaja principal reside en trabajar simuladamente con las complejas y difíciles situaciones reales a través de simplificaciones de la realidad.

\section{Paradigma del cambio social}

Esto supone un cambio tanto al interior como al exterior de la empresa, el primero suele ser consecuencia del segundo. Lo habitual dentro de una empresa, es que las tareas se realicen en consonancia, con las normas y procedimientos de trabajo existentes. (Elorduy Mota, 1993) Es decir, los sistemas de trabajo están fijados de un modo concreto y en el día a día de forma que se siguen manteniendo con la mayor naturalidad.

El cambio (Elorduy Mota, 1993) es, por otro lado, un proceso natural que se produce casi imperceptiblemente aunque no se repare en él ya que es consustancial al transcurso del tiempo. El enfoque teórico de la contingencia (Chiavenato I., 1987) enfatiza que no hay nada absoluto en las organizaciones, todo es relativo y mutable. La organización, en sí misma, un sistema abierto y mantiene transacciones e intercambio con su ambiente (Bustamante, 2004). Esto hace que todo lo que ocurre en el entorno tenga influencia interna sobre lo que ocurre en la organización. De esta manera la viabilidad de una empresa depende del modo en que ésta se enfrenta a las características de a su entorno (Flores, 1994), ya que cada una de ellas puede ser percibida como una oportunidad, un peligro, una limitación o una condición para sus operaciones (Freiberg, et al., 2013).

\section{Paradigma Cultural}

Todas las personas actúan en función de determinadas expectativas respecto de otros y su conducta es social (Widow, 1988). Su entorno, en consecuencia, un conjunto de creaciones humanas y visiones colectivas del futuro, (Gómez, Carlos 1996) las que deben ser asumidas por el individuo, el cual desarrollará su existencia rutinaria de acuerdo con la cultura en que se encuentra inmerso (Flores, 1994).

Aquello que las personas aprenden depende de su sistema cultural, el cual proporciona seguridad y estabilidad respecto del mundo, (Foster, George, 1964) la cultura puede ser definida como la forma común y aprendida de la vida que comparten miembros de una sociedad, y que consta de la totalidad de los instrumentos, técnicas, e instituciones sociales, actitudes, creencias, motivaciones y sistemas de valores que conoce el grupo (Gómez, Carlos 1996).

Cada elemento cultural realiza una función concreta con relación a los otros, y es esencial para la marcha normal de la cultura en general. (Foster, George, 1964) En consecuencia, el entorno cultural de un individuo, grupo u organización debe ser interpretado como un fenómeno integrador.

Así, para bien o para mal, la percepción de la sociedad también cambia y por ende la valoración de sus responsabilidades sociales (Castillo y Parragué, 2008). De esta manera es factible suponer que todo modelo debería considerar una verificación de los parámetros claves del mismo, dado un plazo razonable de tiempo y definiendo un contexto determinado. En este caso, dadas las características y prácticas propias del modelo se consideró un plazo de cuatro años (Freiberg, et al., 2013).

En síntesis, los modelos que ahora se comparan corresponden a la integración de los fundamentos derivados de los tres paradigmas mencionados, se analizan con relación al $\mathrm{Pa}$ radigma Cultural, que le otorga estabilidad y se interpreta con respecto de la dinámica pertinente sobre la base del Paradigma del Cambio Social y finalmente se sostiene de acuerdo a los parámetros derivados de la aplicación del Paradigma Matemático - Estadístico que lo transforma en un modelo formal de medición de la ética empresarial (Battaglia, 2014).

Sobre la base de lo analizado y con el objetivo de resaltar la necesidad de mantener actualizado este modelo de valoración de la contribución social de las empresas a la sociedad (Contardo, 200o), el presente trabajo busca analizar cómo este modelo der responsabilidad social de las empresas (Bustamante, 1996) ha evolucionado a través del tiempo (DeGerencia. com., 26 de Mayo de 2017).

\section{Metodología}

Esta investigación descansa sobre la base de las ideas expuestas en los paradigmas tratados previamente. Su desarrollo se efectuó teniendo en cuenta acciones concretas que se dividieron en las siguientes fases: 
La primera parte, se destinó a la delimitación del universo de empresas e instituciones sociales formalmente organizadas (Hernández, et al, 2006) de la VII Región del Maule, Chile, las que fueron clasificadas en estratos homogéneos considerando el peso relativo de cada una de ellas según el tipo de organización (Bustamante, 1996). Para la determinación de la muestra, se realizó un muestreo aleatorio estratificado proporcional, considerando un $95 \%$ de nivel de confianza y un $5 \%$ de error, además se calculó una varianza a partir de una muestra piloto de organizaciones a fin de que los resultados fuesen proyectados a la población en estudio.

En la segunda etapa, se elaboró un instrumento de recolección de datos que permitiera una eficaz medición de los resultados y que además facilitara la obtención de los datos necesarios para la realización del estudio concluyente (Hernández, et al, 2006). Para ello, se diseñó un cuestionario con las variables previstas para la elaboración del Balance de Responsabilidad Social Empresarial a fin de confirmar la percepción y valoración semántica de los entrevistados respecto de los ítems del cuestionario, el cual fue aplicado sistemáticamente en los dos momentos de investigación denominados aquí modelos inicial y modelo actualizado (Bustamante, 1996, Freiberg, et al., 2013).

La tercera etapa correspondió al análisis del modelo actualizado de Balance de Responsabilidad Social Empresarial respecto del inicial, y para ello fueron consideradas las prioridades asignadas a las variables e indicadores por los distintos agentes de la sociedad consultados, todo lo cual fue medido sobre la base de promedios estandarizados de su importancia relativa

Modelo de Balanc ede
Responsabilidad Social
Empresarial para
Grupo de Variables
Modelo de Balance de
Responsabilidad Social
Empresarial para
Variables
Dónde:
$\mathrm{x}_{\mathrm{i}}=$ Grupos de Variables
$\alpha_{\mathrm{i}}=$ Coeficiente\%

M.B.R.E. $x i=x_{j} i$ $(\alpha \mathrm{i})$

M.B.R.E. $\mathrm{x}_{\mathrm{ii}}=\gamma_{\mathrm{ii}}$

\section{Resultados}

A continuación se presentan las diversas comparaciones derivadas de la formalización de los Modelos de Balance de Responsabilidad Empresarial inicial y actualizado para Grupos en el contexto general de Grupos de variables y Variables (Hernández, et al, 2006).

Los datos estadísticos fueron incorporados a una planilla, se realizó luego un análisis a partir del cálculo de promedios de los coeficientes de prioridad de Grupos de Variables (17), Variables (105); para estandarizarlas ajustando a la normal los valores calculados y, tomando como fundamento la escala Thurstone (1989), se corrigieron los valores estandarizados para obtener valores positivos.

Luego de los análisis previamente descritos se proponen las principales observaciones y derivaciones respecto a los resultados de valores y ponderaciones de las variables y grupos de variables del modelo de Balance de Responsabilidad Social Empresarial (Bustamante, 1996; Chiappe y Consigilio, 2016).

Finalmente, se llevó a efecto el cálculo de porcentajes, totalizados al 100\%, de los promedios estandarizados, por supuesto, expresados en términos positivos para la obtención de coeficientes que darían cuerpo al Modelo Matemático del Balance de Responsabilidad Social Empresarial (Freiberg, et al., 2013) y que representan la importancia relativa asignada, por parte de los agentes sociales consultados, a los distintos Grupos de Variables y Variables dentro del modelo en estudio (Castillo y Parragué, 2008).

\section{Notación y Significados}

La notación y significado a los distintos parámetros utilizados en el modelo alcanza una sumatoria de coeficientes porcentuales que llega a 1, equivalente al 100\% de las prioridades estandarizadas como se indica en las formulaciones 1 y 2 .

$$
\begin{array}{lll}
\sum_{\boldsymbol{i}=\mathbf{1}}^{\mathbf{n}} \alpha \mathrm{i} & \mathrm{x} & \mathrm{n}=17 \\
\sum_{\boldsymbol{i}=\mathbf{1}}^{\mathbf{n}} \beta_{\mathrm{ii}} & \gamma & \mathrm{i}=1 \\
& \mathrm{n}=103-105
\end{array}
$$

$$
\begin{aligned}
& \gamma_{\text {ii }}=\text { Variables } \\
& \beta_{\text {ii }}=\text { Coeficiente } \%
\end{aligned}
$$

de variables, Variables y Factores de Componentes principales.

Modelo de responsabilidad empresarial para grupos de variables

Este primer análisis se obtuvo a partir de los coeficientes promedios de los valores de cada 
grupo de variables cada una de las cuales posee sus respectivos coeficientes en porcentajes de las medias estandarizadas para los grupos de variables (Bustamante, 1996).

En general la sociedad considera muy importante la gestión tanto interna como externa de la empresa, otorgándole especial atención a 11 de estos 17 grupos de variables, destacándose entre los que más preocupan los grupos referidos a la Situación económica y financiera, Situación comercial, Seguridad y, Medio ambiente y ecología.

La sociedad necesita de una gestión que involucre tanto la responsabilidad de lo que produce la empresa, como así mismo, cómo lo hace y a quienes involucra y en quienes impacta este proceso de generación de riqueza. En cuanto a la desviación estándar de los grupos, esta alcanza valores marginales lo cual implica que la dispersión de los juicios emitidos por la sociedad es baja. Cabe señalar que el grupo de Situación económica y financiera $\mathrm{C} 1$, es el que posee una menor dispersión en las respuestas.

En general, la interpretación del modelo para grupos de variables indica que se ha mantenido casi invariable para los modelos inicial y actualizado, mostrando algunas diferencias en las variables de los Grupos Gi Situación Económico Financieras y G2 Situación Comercial en que las medias se ubican por encima de las medias del modelo inicial.

Por su parte, los grupos a los que se les asigna una menor importancia en el modelo actualizado son: Participación y comunicación,
Relación con la comunidad y Compensaciones e incentivos. Sin embargo, todos ellos están clasificados, en general, dentro del rango de importantes para la sociedad.

Sin perjuicio de lo indicado con relación a la escasa varianza de los datos, se procedió a estandarizar las medias de los Crupos y a calcular los pesos relativos de estos dentro del modelo. La tabla 1 muestra los resultados coeficientes estandarizados y en porcentajes de los modelos matemáticos puestos en contraste.

Como se puede observar, en el Modelo inicial, el grupo de variables G6 Servicio al Cliente con un coeficiente de 10,2 puntos porcentuales fue el calificado con mayor prioridad, poniendo de manifiesto la relevancia de la orientación externa y comercial de las empresas que según las organizaciones sociales debe ser atendida dado el impacto o externalidad que representa para la sociedad en su conjunto. Por otra parte, el grupo de variables G2 Situación Comercial que con un coeficiente de 2,3 puntos porcentuales era la variable con menor relevancia en el contexto general.

El Modelo actualizado por su parte muestra que el grupo de variables C17 Relaciones con la Comunidad con un coeficiente de 11,37 puntos porcentuales fue el calificado con mayor prioridad, poniendo de manifiesto una preocupación relevante y eventualmente una carencia que, según las organizaciones sociales, debe ser atendida dado el impacto que representa para la sociedad en su conjunto.

Tabla 1: Contraste de los modelos inicial- actualizado de responsabilidad social empresarial para grupos de variables

\begin{tabular}{|c|c|}
\hline Modelo matemático inicial & Modelo matemático actualizado \\
\hline 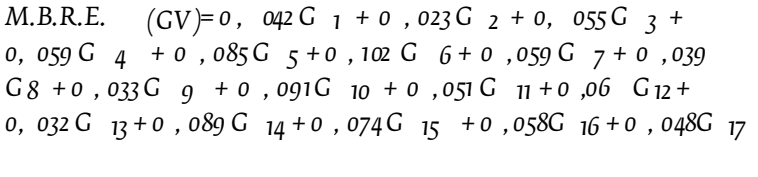 & 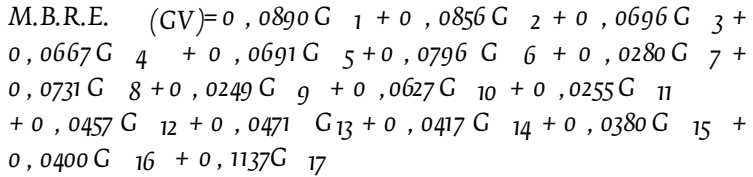 \\
\hline
\end{tabular}

Por otra parte, el grupo de variables G9 Compensaciones e incentivos con un coeficiente de 2,49 puntos es la variable menos relevante para la sociedad, lo que puede ser originado por tratarse de un aspectos suficientemente cubierto por las normas legales vigentes.

Analizados en conjunto, los modelos inicial - actualizado, se observan diferencias de magnitud, marcándose con nitidez las distancias entre los grupos Gi Situación Económico Financiera y G2 Situación Comercial, lo mismo ocurre al el grupo G17 Amenazas a la Salud de la Población, que el año 2000 cuenta con dos nuevas variables por encima de las que poseía en año 1996, ubicándose con una media estandarizada superior del orden de un $11,37 \%$, en tanto que el año 1996 alcanzó sólo un 4,8\%, poniendo de manifiesto preocupaciones medio ambientales con relación a su impacto en las personas, actitud concordante con lo observado global- mente en las economías más desarrolladas.

Modelo de responsabilidad social empresarial actualizado para variables

Los coeficientes estandarizados de cada una de las 105 variables que contempla el modelo fueron promediados y estandarizados a fin de calcular los valores porcentuales y estructurar los pesos relativos y según estos valores, orientar las decisiones de las empresas con el propósito de enfocar la gestión hacia aquellas variables que mejor se ajusten a las demandas sociales.

El Modelo inicial muestra que la variable más relevante resultó ser Calidad con una media de 9,706 puntos del Grupo G3 Producción, al mismo tiempo, la Variable que alcanzó la más baja prioridad fue Ventas del Grupo G2 Situación Comercial con 8,390 puntos.

Por otra parte, el Modelo actualizado presenta como variable más relevante la Rentabi- 
lidad del Grupo Gı Situación Económico Financiera con una media de 9,464 puntos dado el impacto que presenta para las empresas y para la sociedad en su conjunto. Por otra parte, el Grupo de variables $\mathrm{G}_{7}$ de Relaciones con la Comunidad es la que presenta la variable Imagen de la Empresa como la menos importante con una media de sólo 8,360 puntos.

Sobre la base del modelo de balance actualizado, (Bustamante, 1996) variables tales como calidad, imagen de la empresa, calidad del producto, satisfacción del consumidor, amenazas a la salud de la población y contaminación del medio ambiente, se refieren inequívocamente a la relación de la empresa con su entorno y sus clientes. Importante se torna entonces que los consumidores perciban que la empresa se preocupa no sólo por entregar un producto o un servicio, sino que esto se realice de la mejor forma.

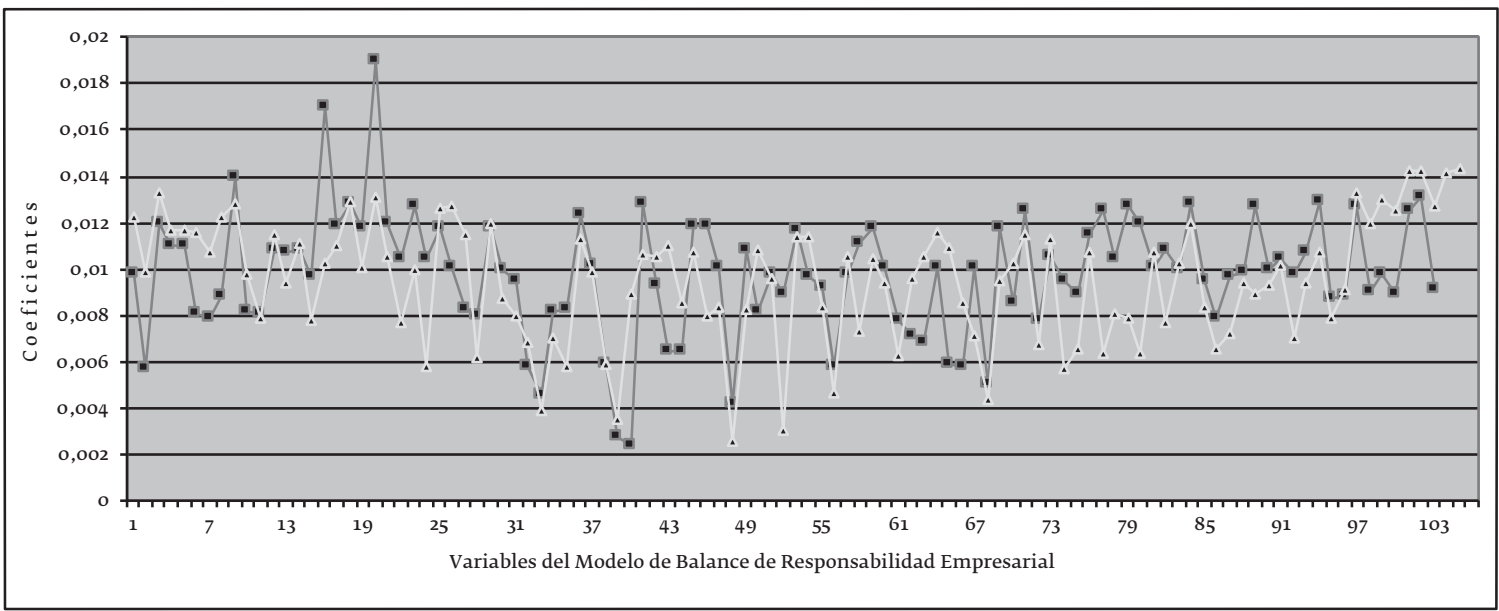

Figura 1: Evolución del modelo de balance de responsabilidad empresarial para variables

Del mismo modo, variables como rentabilidad, ventas, productividad económica y rentabilidad de la empresa, no dejan de ser indiferentes para la sociedad; puesto que la empresa es fuente de trabajo y a la vez entrega sus productos a la sociedad de manera que su existencia depende del grado de atención que demuestre para con los diversos agentes de la sociedad. Cabe destacar también, que las dos nuevas variables incorporadas al Modelo actualizado recibieron una calificación de muy importantes para la sociedad. Así, Reciclaje recibió una puntuación promedio de 9,4 puntos; mientras que la variable Renovación de recursos naturales explotados recibió un promedio de 9,8 puntos incorporándose al Grupo de Variables G17 Medio Ambiente y Ecología.

Los valores estandarizados de las variables muestran que la mayor prioridad es para la variable Ahorro de Energía (G17V105) con un coeficiente de 0.0143 puntos, perteneciente al Grupo de Variables 17 Medio Ambiente y Ecología, lo que pone de manifiesto las restricciones energéticas vividas el último año. Por el contrario la variable (G11V48) Revista de Información Interna presenta un coeficiente de sólo 0.0026 puntos y pertenece al Grupo de Variables G11 Participación y Comunicación.

A este nivel de la interpretación, poca duda cabe de lo razonable de los resultados que explicitan con claridad los eventos globales que afectan a todas las economías, como son los temas energéticos y del medio ambiente, perdiendo prioridad esfuerzos de los años 70 como la edición de medios de comunicación interna de las empresas y los relacionados con la participación y comunicación, que en la actualidad parecen ser consustanciales a la gestión racional de las empresas.

\section{Modelo de factores de componentes principales, grupos de variables}

Sobre la base del Paradigma del Cambio Social y apoyado en el Paradigma Cultural se consideró pertinente actualizar el modelo cada cuatro años, lo que redundó en el descubrimiento de dos nuevas variables para medir la responsabilidad empresarial, las que, como ya se mencionó, se agregaron a la dimensión ambiental del Grupo 17 de variables del Modelo, afectando sus medias estandarizadas e importancia relativa.

A fin de profundizar el análisis, la tabla 2 presenta los modelos de prioridad social según el Análisis de Factores de Componentes Principales, de acuerdo con los datos antes mencionados, considerando una expresión matemática del énfasis de preocupación social que significa para los agentes sociales cada factor y el detalle de cada uno de sus componentes principales, especificando los Grupos de Variables relevantes que los constituyen y las Varianzas Totales Explicadas por cada modelo. 
Tabla 2: Modelos de factores de componentes principales del balance de responsabilidad social empresarial, para grupos de variables inicial - actualizado

\begin{tabular}{|c|c|}
\hline Modelo matemático de balance social inicial & Modelo matemático de balance social actualizado \\
\hline 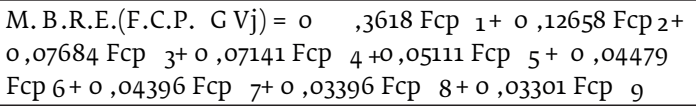 & 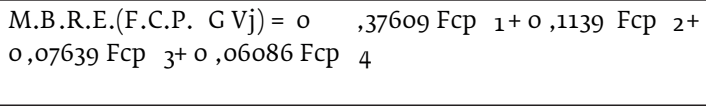 \\
\hline Varianza Total Explicada : $\quad 84,484$ & Varianza Total Explicada : \\
\hline
\end{tabular}

Los datos obtenidos demuestran que el Modelo inicial explica una varianza de $84,5 \%$ en tanto que el Modelo actualizado explica sólo un $62,7 \%$, esto debido a que el primero cuenta con 9 Factores y el segundo con sólo 4 Factores, sin embargo si se totaliza la varianza explica de los cuatro primeros factores del Modelo 1996, esta alcanza a $63,7 \%$ lo que hace a los modelos, en general, equivalentes.

Efectivamente los cuatro primeros factores resultan perfectamente comparables en valor no así en contenido cuyos componentes son efectivamente diferentes y representativos de dos realidades económico - sociales que se expresan de acuerdo con las preocupaciones de cada momento. El Anexo 6 contie- ne un detalle de los Factores y de los Componentes Principales determinados para ambos Modelos de Responsabilidad Empresarial.

\section{Modelo de factores de componentes principales para variables}

Con el propósito de ampliar el análisis en los elementos principales de la Responsabilidad Empresarial, se procedió al cálculo de los coeficientes matemáticos de las variables determinadas sobre la base de un estudio de Factores de Componentes Principales, determinando un modelo de Factores que se presenta en la tabla 3.

Tabla 3: Modelos deModelos de factores de componentes principales del balance de responsabilidad social empresarial, para variables inicial - actualizado

\begin{tabular}{|c|c|}
\hline Modelo inicial & Modelo actualizado \\
\hline $\begin{array}{l}\text { M.R.E.(F.C.P.V v j) }=0,22724 \mathrm{Fcp}_{1}+\mathrm{o}, 08098 \mathrm{Fcp}_{2}+ \\
\text { o,o586 Fcp }+ \text { o,o4468 Fcp } 4+0,03758 \mathrm{Fcp}_{5}\end{array}$ & 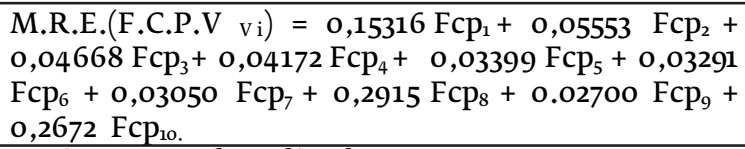 \\
\hline Varianza Total Explicada: 44,908 & Varianza Total Explicada: 47,736 \\
\hline
\end{tabular}

Por otra parte y, en el mismo sentido del modelo mencionado, el modelo determinado el año 1996 permitió identificar cinco factores que permitieron explicar un $44,908 \%$ de la Varianza Total como se muestra en el recuadro siguiente que sintetiza las preocupaciones sociales de la sociedad organizada en la forma de una demanda ordenada hacia el sector empresarial. Las variables que exponen cualitativamente dichas orientaciones claves se detallan en el anexo 3. Un modelo similar fue elaborado para las variables de responsabilidad empresarial mediante el uso del análisis de factores de componentes principales, mediante el cuál se pudo identificar para el año 2000, diez factores relevantes, que lograron explicar el $47.736 \%$ de la varianza total.

Se observa en las cifras que los componentes principales de los factores determinados presentan coeficientes similares a los del modelo de variables en general. Por lo que resulta conveniente, entonces, considerarlo en la evaluación de la gestión de la empresa, puesto que permite concentrar el análisis, otorgando el énfasis necesario a las principales variables de preocupación social, representadas en los Componentes Principales.

\section{Discusión}

Del análisis de los modelos de balances (Bustamante, 1996) se aprecia, de manera global en la tabla 4, el comportamiento de los grupos de variables más utilizados, destacándose en primer lugar el grupo Situación Económico Financiera, con un 92\% de frecuencia, Situación Comercial con un $78 \%$ y Servicio al Cliente con un $77 \%$, como se puede observar en la siguiente tabla.

Por otro lado, los grupos de variables menos utilizados fueron Relaciones con la Comunidad con sólo un $54 \%$, Personal con un $61 \%$ y Productividad con un $60 \%$.

Las variables más usadas y, por ende, de mayor relevancia para el personal de las empresas, son Seguridad del empleo, Relaciones laborales, Capacitación y desarrollo, Fondos de salud. Por otra parte, las variables de Responsabilidad Empresarial más relevantes desde el punto de vista de los ejecutivos de las empresas, fueron Seguridad de empleo, Accidentes de trabajo, Enfermedades profesionales, Trabajo temporal y Sistema de remuneraciones. 
Con relación a la jerarquización de variables de responsabilidad social empresarial desde la perspectiva de la sociedad, la variable más im- portante para la sociedad resultó ser Satisfacción Laboral y, la menos relevante resultó ser Trabajo Temporal.

Tabla4: Análisis del modelo actualizado

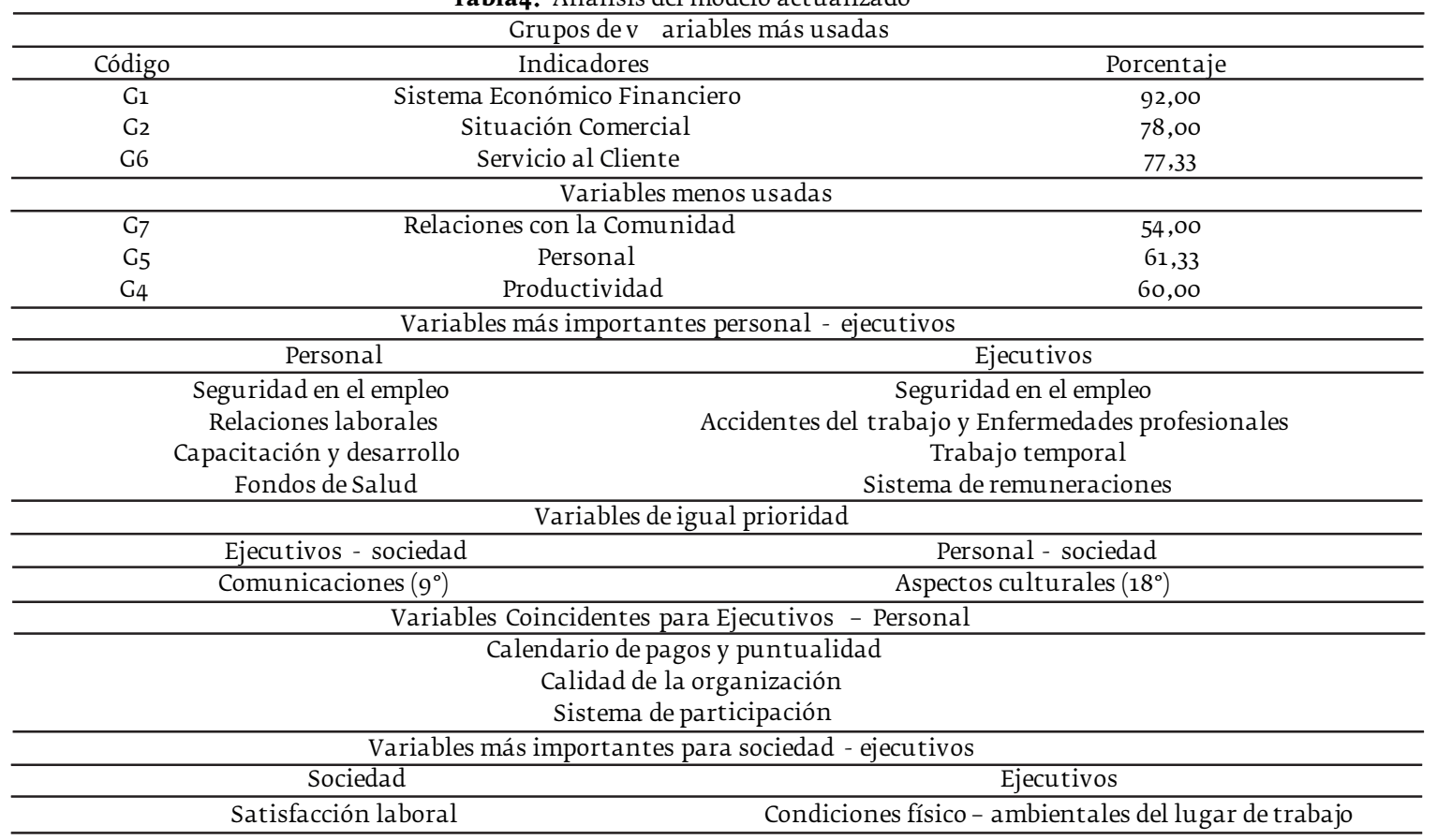

Del análisis comparativo de las prioridades de variables desde el punto de vista del personal y de los ejecutivos v/s la sociedad, se puede concluir que existe un mayor grado de similitud entre las prioridades de las variables de Responsabilidad Empresarial, entre las seleccionadas por el personal con las seleccionadas por la sociedad. Así se puede decir que el personal de las empresas está más cerca que los ejecutivos de la priorización dada por la sociedad en su conjunto (Widow, 1988).

La variable de Responsabilidad Empresarial que presenta la misma prioridad tanto para los ejecutivos como para la sociedad es "Comunicaciones", la cual ocupa, en ambos casos, el noveno lugar de importancia. En tanto que la variable de Responsabilidad Empresarial que posee el mismo grado de importancia, tanto para la sociedad como para el personal de la empresa, es "Aspectos Culturales”, ubicándose en el lugar número 18.

A pesar de mostrar una mayor dispersión de datos, con desviación de 5.76 puntos, el análisis comparativo de los ejecutivos con el personal de la organización, presenta 3 variables que coinciden en sus prioridades, estas son "Calendarios de pagos y puntualidad", en el sexto lugar de importancia, "Calidad de la organización”, ocupando el número siete y "Sistema de participación", ubicado en la décima segunda prioridad.

Para la sociedad por su parte, la variable más relevante fue Satisfacción Laboral, con la mayor puntuación, en tanto que los ejecutivos en su lugar asignan relevancia a la variable Condiciones físico - ambientales del lugar de trabajo, se puede concluir entonces que los ejecutivos de las empresas no consideran como relevante una variable que para la sociedad si lo es.

\section{Conclusiones}

El presente estudio consiguió analizar la evolución del modelo de balance de responsabilidad empresarial para grupo de variables y variables y obtuvo dos nuevas variables, "fondos utilizados en reforestación" y "Reciclaje y conservación del ambiente", sugerido por las organizaciones consultadas, incluidas entre las variables del grupo renovación de recursos naturales.

Es de esperar que la validación del modelo haya permitido incentivar a las organizaciones con respecto al tema, poniendo de manifiesto que el balance propicia beneficios en forma integral lo que se verá reflejado en todos los ámbitos de su quehacer empresarial. Así quedó de manifiesto a lo largo de la investigación que el balance social, al registrar las variables elegidas por cada empresa, 
puede contribuir primero a destacar y después a mejorar los aspectos negativos de la organización; asimismo puede dar origen, a mejoras paralelas en otras variables y a la vez a partir de éstas sobre otras de las variables del modelo.

Además el balance social, como aportación metodológica, podría facilitar la planificación, participación y el auto control de las organizaciones, contribuyendo con eficacia a un proceso estratégico coherente puesto que les permita iniciar un proceso de adaptación al entorno lo cual pondría en movimiento los recursos tecnológicos, financieros y principalmente los talentos humanos de sus personales.

\section{Referencias}

Amato, C. N. (2015). Relación entre sustentabilidad, responsabilidad social y responsabilidad extendida al productor. Argentina: Consejo Nacional de Investigaciones Científicas y Técnicas. Recuperado de https://sustainabledevelopment. un.org/content/documents/639491-Amato-Relacion\%2oentre $\% 20$ Sustentabilidad $\% 20$ Responsabilidad $\% 20$ Social $\% 20 y \% 20$ Responsabilidad $\% 20$ Extendida $\% 20$ al\%2oProductor.pdf

Antonio Freije, " La utilización de modelos de valoración social permitirá comparar entre sí diversas organizaciones" Estrategía y Políticas de Empresa,Ediciones Deusto S.A., $3^{\underline{a}}$ edición, España, 1996.

Aristóteles, Política, Libro I Capítulo 2, Editorial Panamericana, Santa Fe de Bogotá, Colombia, 1992, "Sólo una bestia o un Dios puede vivir fuera de la polis..."

Battaglia, M.; Testa, F.; Bianchi, L.; Iraldo, F.; Frey, M. (2014). Corporate Social Responsibility and Competitiveness within SMEs of the Fashion Industry: Evidence from Italy and France. In Sustainability, pp. 872-893.

Bustamante U. Miguel A., Opazo B. Pablo A. 2004. Hacia Un Concepto De Complejidad: Sistema, Organización Y Empresa. Serie Documentos Docentes FACE SDD, año 2, $\mathrm{N}^{\circ} 3.23 \mathrm{P}$.

Bustamante, Miguel, Elaboración de un Modelo de Balance de responsabilidad Empresarial para las Empresas de la Séptima región del Maule, Chile, Tesis Doctoral, universidad de Deusto, 1996.

Capra Fritjof, El punto crucial, ciencia, sociedad y cultura naciente. Simon \& Schuster. New York.1982.

Castillo, P. C., \& Parragué, P. M. (2008). Responsabilidad Social Empresarial . Universidad de Chile, Control de Gestión y Sistyemas Informaticos, Facultad de Economía y Negocios, Santiago.

Chevalier, Alain, Le bilan social de l'entreprice, Masson S. A. 1976, París, Francia Y Gelinier, Octave, Estrategia Social de la Empresa, Editions Hommes et Techniques, Asociación para el progreso de la Dirección, 1976, Madrid, 1978.

Chiappe, I. S., \& Consigilio, L. (16 de Agosto de 2016). É Revistas Logisticas . Obtenido de ¿Qué implica la RSE en la Actualidad ?: http://www.logisticasud.enfasis.com/articulos/75745-que-implica-la-rse-la-actualidad

Chiavenato Idalberto, Introducción a la Teoría General de la Administración, Editorial McGraw- Hill Latinoamericana S.A., $3^{\underline{a}}$ Edición , Bogotá, Colombia, 1987.

Chiavenato, "Modelo es la representación de alguna cosa o el estándar de algo a ser hecho. A través de él es posible hacer representaciones de la realidad." Op. Cit.

Chumacher, Edward. F., Lo pequeño es hermoso, Madrid. Orbis, 1983.

Contardo, Cecilia Y Leticia Pérez, Memoria de Grado, Modelo De Balance De Responsabilidad Empresarial Una Visión Al 20oo, Prof. Guía M. Bustamante, Ing. Comercial U. De Talca, Chile.

David P. Baron, Agus Harjoto, Jo Hoje. The Economics and Politics of Corporate Social Performance. Business and Politics. August 2011 (a), Vol. 13, Issue 2, Pages 1-48.

DeGerencia.com. (26 de Mayo de 2017). De Gerencia.com. Obtenido de Las nuevas acciones que Revolucionan la RSE: http://www.degerencia.com/noticia/11740o/las-nuevas-acciones-que-revolucionan-la-rse

Elorduy Mota, Juan Ignacio, Estrategia de Empresa y Recursos Humanos: Una Visión Dinámica de la Empresa, Editorial McGraw-Hill Interamericana de España S.A., $1^{\underline{a}}$ Edición, Madrid, España, 1993.

Escala Thurstone o Método de Intervalos de igual apariencia, objetivo es obtener una escala unidimensional con propiedades de intervalos. Con esto se conservan las distancias originales. Aaker y Day, 1989, Op. Cit..

Flores, Fernando, en el contexto del Seminario: La Empresa Emergente y la Sociedad del Futuro. págs. 3 - 6, Santiago de Chile, 1994.

Foster, George, Las Culturas Tradicionales y los Cambios Técnicos, Fondo de Cultura Económica, $3^{\text {a }}$ Edición, Mexico, 1964.

Freiberg H. A., J. B. Stover, G. de la Iglesia, M. Fernández, (2013) Polychoric And Tetrachoric Correlations In Exploratory And Confirmatory Factorial Studies. Prensa Médica Latinoamericana - ISSN 1688-4094 Ciencias Psicológicas, VII (2): $151-164$

Friedman, Milton, Capitalismo y Libertad, Ediciones Mialp S.A., México,1988.

Gómez, Carlos, El factor Cultural en la Administración de Empresas, Revista Gestión, Año XXI, № 254, Mayo 1996, pág. 16.

Hernández, R., Fernández, C. y Baptista, P. Metodología de la Investigación. México D. F.: Mc Graw-Hill (2006)

Hiil, C.W. \& Jones, G. R. (2005). Administración Estratégica, Un Enfoque Integrado, Sexta Edición, Mc Graw Hill, México.

Juan I. Elorduy, "El Cambio se define como cualquier modificación observada, que permanece con un carácter relativamente estable". Op. Cit.

Parra Luna, Francisco. 1980. Balance social y progreso empresarial (aplicacion de la teoria de sistemas a un ejemplo cifrado de balance social de la empresa). Madrid : Cirde, D.L., 1980.

Quispe, D. A., Castellanos, I. O., Valenzuela, J. E., \& Villanueva, L. A. (2015). Responsabilidad Socail Empresarial (RSE): Torías, Tendencias 
y Desafíos futuros para Nolivia. Bolivia: Konrad Adenauer Stiftung e.V. Obtenido de http:// www.kas.de/wf/doc/22089-1442-4-30.pdf

Rodríguez Luño, Angel, "Etica” Libro II Capítulo 1, Ediciones Universidad de Navarra S.A., $2^{\underline{a}}$ Edición, Pamplona, 1984.

Rosa, Y. R. (3o de mayo de 2013). EOI: Escuela de Organizacion Industrial, Master Executive en Direccion de Empresas Tecnológicas e Industriales. Recuperado de http://www.eoi. es/blogs/mintecon/2013/o5/3o/el-conceptode-rse-desafios-y-oportunidades/

Satién, María Luisa, Indicadores Sociales de Calidad de Vida, un sistema de medición aplicado al Pís Vasco, Centro de Investigaciones Socio- lógicas, Siglo XXI de España Editores S. A., Madrid, España, 1993.

Verdugo Marinkovic, Mario y Ana María García Barzelatto, Manual de derecho político, Tomo I, Edición Jurídica de Chile, 2 ${ }^{\mathrm{a}}$ Edición, 1998.

Vohrer, M. (1998), De la economía social de mercado a la economía ecológica de mercado, Perfiles Liberales, No. 62, pp. 16-29.

Widow, Juan Antonio, El Hombre Animal Político: Orden Social, Principios e Ideologías, Tercera parte, Capítulo III, Editorial Universitaria, $\mathbf{2}^{\underline{a}}$ Edición, Santiago, Chile, 1988. 